\title{
Veillonella rogosae sp. nov., an anaerobic, Gram- negative coccus isolated from dental plaque
}

\author{
Nausheen Arif, ${ }^{1}$ Thuy Do, ${ }^{1}$ Roy Byun, ${ }^{2}$ Evelyn Sheehy, ${ }^{1}$ Douglas Clark, ${ }^{1}$ \\ Steven C. Gilbert ${ }^{1}$ and David Beighton ${ }^{1}$
}

Correspondence

David Beighton

david.beighton@kcl.ac.uk

\author{
${ }^{1}$ Department of Microbiology, The Henry Wellcome Laboratories for Microbiology and Salivary \\ Research, King's College London Dental Institute, Floor 17, Guys Tower, London Bridge, \\ London SE1 9RT, UK \\ ${ }^{2}$ Institute of Dental Research, Westmead Centre for Oral Health and Westmead Millennium \\ Institute, Westmead Hospital, Wentworthville, NSW 2145, Australia
}

At the time of writing, the genus Veillonella comprised the following recognized species: Veillonella parvula, V. atypica, $V$. dispar, $V$. criceti, $V$. ratti, $V$. rodentium, $V$. caviae, $V$. montpellierensis and $V$. denticariosi (Mays et al., 1982; Rogosa, 1984; Jumas-Bilak et al., 2004; Byun et al., 2007). Members of the genus are non-fermentative, anaerobic, Gram-negative, small cocci isolated from the oral cavity and intestinal tract of humans and animals that gain energy from the utilization of short-chain organic acids (Delwiche et al., 1985). Species of the genus isolated from humans are $V$. parvula, V. atypica, $V$. dispar, $V$. montpellierensis and $V$. denticariosi, although a strain described as a member of the $V$. ratti-V. criceti group has been found in a semen sample from a patient attending for treatment of infertility at a urology unit (Marchandin et al., 2005). The above species may be associated with monomicrobial infections in humans (Marchandin et al., 2001).

Identification of taxa to the genus Veillonella is quite straightforward but the identification of members of the genus to the species level is difficult owing to the lack of

\footnotetext{
The GenBank/EMBL/DDBJ accession numbers for the 16S rRNA gene sequences of strains CF100 ${ }^{\top}, \mathrm{CF} 05, \mathrm{CF} 30, \mathrm{CF} 84, \mathrm{CF} 88$ and CF24 are EF108443, EF108444, EF108445, EF108446, EF108447 and EF108448, respectively, and that for the partial rpoB gene sequence of strain $\mathrm{CF} 100^{\top}$ is EF2 11831 .
}

discriminating phenotypic tests (Kolenbrander \& Moore, 1992). Analysis of 16S rRNA gene sequences permits the identification of isolates as members of the genus Veillonella. However, very low levels of $16 \mathrm{~S}$ rRNA gene sequence dissimilarity are found within the genus and, although $V$. atypica, $V$. montpellierensis and the species isolated from rodents are identifiable by this means, discrimination between $V$. dispar, $V$. parvula and $V$. denticariosi is problematic (Jumas-Bilak et al., 2004; Byun et al., 2007). Additionally, there have been frequent reports of the occurrence of intra-chromosomal heterogeneity between 16S rRNA genes in human isolates of Veillonella species, further complicating the identification of isolates on the basis of $16 \mathrm{~S}$ rRNA gene sequencing (Marchandin et al., 2003). In order to overcome the limitations associated with using 16S rRNA gene sequences to identify Veillonella species, comparisons of partial $d n a K, r p o B$ and $g y r B$ gene sequences have been proposed as a reliable method to identify members of the genus. All recognized members of the genus can be discriminated by using dnaK gene sequence comparison and, in particular, V. parvula and $V$. dispar have been reliably identified in this way (JumasBilak et al., 2004). In a more recent report, the ability of dnaK gene sequencing to differentiate between Veillonella species, including $V$. denticariosi, was confirmed, and comparisons of $r p o B$ and $g y r B$ gene sequences were also shown to enable the identification of species within the 
genus, with the $r p o B$ gene being the more discriminatory (R. Byun, personal communication).

As part of a large study investigating the genotypic diversity of Veillonella species present in the dental plaque of cariesfree children and in the infected dentine of carious lesions, we have frequently isolated strains that appeared based on $16 \mathrm{~S}$ rRNA gene sequencing to be distinct from all recognized species of the genus. The aim of the present study was to determine the taxonomic status of these isolates by using a polyphasic approach.

Occlusal or buccal surface plaque was collected from nine caries-free children. The children or their carers gave consent to the collection of the samples and the study was approved by the local ethics committee (ref. 02/11/10). Veillonella-like isolates were recovered on agar consisting of (per litre deionized water) $5 \mathrm{~g}$ Bacto tryptone (Difco), $5 \mathrm{~g}$ Bacto yeast extract (Difco), $0.75 \mathrm{~g}$ sodium thioglycolate (Sigma), $0.002 \mathrm{~g}$ Bacto basic fuchsin (Difco), $21 \mathrm{ml} 60 \%$ sodium lactate (Sigma) and $15 \mathrm{~g}$ Bacto agar (Difco). The $\mathrm{pH}$ was adjusted to 7.5 prior to autoclaving; vancomycin (7.5 $\mu \mathrm{g} \mathrm{ml}^{-1}$; Sigma) was added following autoclaving (Rogosa, 1956). Inoculated plates were incubated in an atmosphere consisting initially of $90 \%(\mathrm{v} / \mathrm{v})$ nitrogen, $5 \%$ $(\mathrm{v} / \mathrm{v})$ hydrogen and $5 \%(\mathrm{v} / \mathrm{v})$ carbon dioxide at $37{ }^{\circ} \mathrm{C}$ for 4 days. Isolates were presumptively identified as members of the genus Veillonella on the basis of their ability to grow on the selective isolation medium and their typical colonial appearance: colonies were $2-4 \mathrm{~mm}$ in diameter, regular and slightly domed in shape with an entire edge.

To confirm the identity of the isolates as belonging to the genus Veillonella, genomic DNA was obtained from each isolate by suspending colonies in deionized water and heating the cells to $95{ }^{\circ} \mathrm{C}$ for $10 \mathrm{~min}$. The $16 \mathrm{~S}$ rRNA gene was amplified by using the primers $27 \mathrm{f}$ and 1492r (Lane, 1991) and sequencing was performed by using the $27 \mathrm{f}$ primer in conjunction with Big Dye Ready Reaction Termination Mix (ABI) to obtain short 16S rRNA gene sequences of 450-500 nt. These sequences were submitted to the Ribosomal Database Project (http://rdp.cme. msu.edu/) via the Sequence Match routine. The majority $(>99 \%)$ of isolates were identified as members of the genus Veillonella.

The partial $16 \mathrm{~S}$ rRNA gene sequences $(450-500 \mathrm{nt})$ of all the new isolates were aligned by using CLUSTAL W (Thompson et al., 1994) in BioEdit (http://www.mbio. ncsu.edu/BioEdit/bioedit.html) with partial 16S rRNA gene sequences of $V$. caviae DSM $20738^{\mathrm{T}}, V$. rodentium ATCC $17743^{\mathrm{T}}$, V. montpellierensis ADV $281.99^{\mathrm{T}}$, V. parvula ATCC $10790^{\mathrm{T}}$, V. dispar ATCC $17748^{\mathrm{T}}$, V. atypica ATCC $17744^{\mathrm{T}}$, V. ratti ATCC $17746^{\mathrm{T}}$, V. criceti ATCC $17747^{\mathrm{T}}$ and $V$. denticariosi CIP $109448^{\mathrm{T}}$. Phylogenetic relationships between taxa were analysed by using MEGA 3.1 (Kumar et al., 2004). Distances were calculated according to the Kimura two-parameter model and clustering was based on the neighbour-joining method of Saitou and Nei (1987) by using bootstrap values based on 1000 replicates. From these analyses, a group of isolates was identified that appeared to represent a novel taxon that was distinct from all other clinical isolates and from other type and reference strains investigated (data not shown).

In order to understand the phylogenetic position of these isolates more clearly, more complete $16 \mathrm{~S}$ rRNA gene sequences (1355 nt) were obtained from single strains (designated $\mathrm{CF} 100^{\mathrm{T}}, \mathrm{CF} 05, \mathrm{CF} 30, \mathrm{CF} 84, \mathrm{CF} 88$ and CF24), each isolated from a different, unrelated subject. The $16 \mathrm{~S}$ rRNA gene sequences of these six strains, and also of $V$. parvula ATCC $10790^{\mathrm{T}}$, exhibited a high level of sequence similarity with many cloned 16S rRNA gene sequences from taxa isolated from the human mouth or gut (data not shown). Alignment of the longer 16S rRNA gene sequences revealed similarity of $>99.3 \%$ (mean $99.7 \%$ ) among the six novel strains, demonstrating that they belonged to the same species. They showed the highest 16S rRNA gene sequences similarity to $V$. parvula ATCC $10790^{\mathrm{T}}$ (98\%). Phylogenetic relationships between the new isolates and recognized species of the genus Veillonella were investigated as described above with Dialister pneumosintes ATCC $33048^{\mathrm{T}}$ as the outgroup organism. The resulting neighbour-joining tree is shown in Fig. 1. When the 16S rRNA gene sequence data were analysed by using the minimumevolution method in MEGA 3.1 (Kumar et al., 2004), the resultant tree had the same appearance with the novel strains on a distinct branch separated from other Veillonella species with a bootstrap value of $32 \%$. When the interior branch test of phylogeny (Kumar et al., 2004) was employed to test the significance of the branch containing the six novel strains, the confidence probability obtained with the neighbour-joining and minimumevolution methods was 84 and $88 \%$, respectively, indicating that discrimination between $V$. parvula and the novel strains was approaching significance. However, $16 \mathrm{~S}$ rRNA gene sequence analysis does not reliably distinguish between Veillonella species (Jumas-Bilak et al., 2004; Byun et al., 2007).

To overcome the above difficulty, comparisons of dnaK, gyrB and $r p o B$ gene sequences have been used to differentiate between Veillonella species, including $V$. denticariosi (Jumas-Bilak et al., 2004; Byun et al., 2007). We used this approach in this study to determine the position of the six novel strains more accurately. No amplicons were produced when we used the primers previously described for the $d n a K$ gene (Jumas-Bilak et al., 2004). We therefore used primers for the rpoB gene (VeillrpoBF, GTAACAAAGGTGTCGTTTCTCG; Veill-rpoBR, GCACCRTCAAATACAGGTGTAGC) and were successful in obtaining and sequencing amplicons from all the strains investigated. The sequences (608 nt) were aligned, as described above, with the $r p o B$ gene sequences of $V$. parvula strains ATCC $10790^{\mathrm{T}}$, ATCC 17745, RBV162, RBV167, RBV180, RBV182, RBV175, RBV30, RBV123, RBV156, RBV173, RBV184, RBV54, RBV186, RBV188, RBV35, RBV136, RBV192, RBV183, RBV159, RBV146, RBV78, RBV179 and RBV185, V. atypica ATCC $17744^{\mathrm{T}}, V$. 


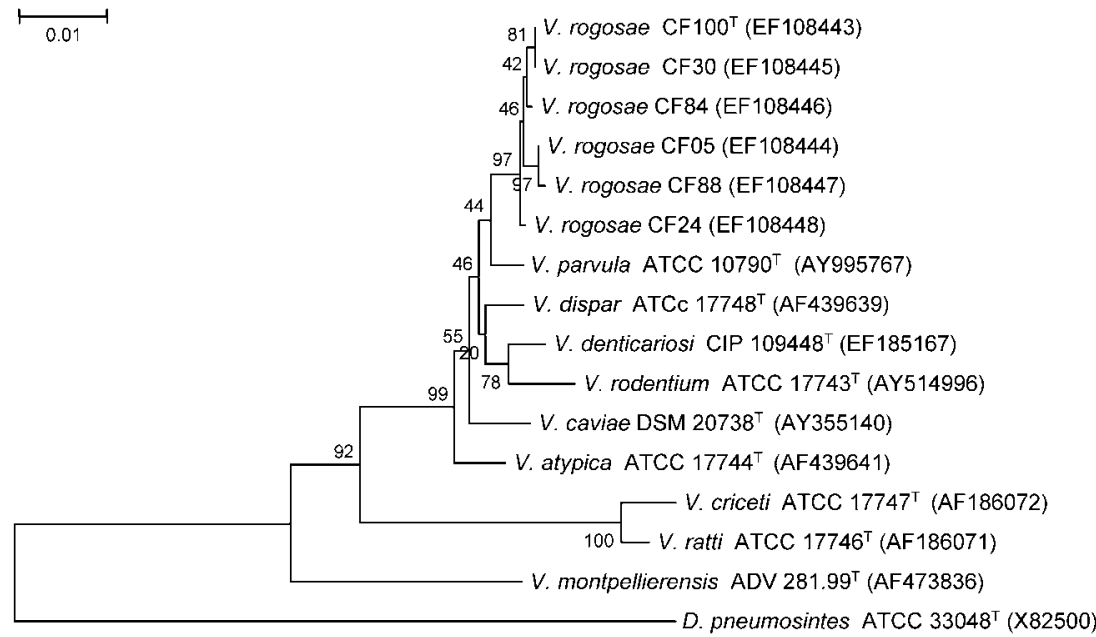

Fig. 1. Neighbour-joining tree based on nearly complete 16S rRNA gene sequences (1309 nt), showing relationships between strains $\mathrm{CF}_{100}{ }^{\top}, \mathrm{CF} 05, \mathrm{CF} 30, \mathrm{CF} 84, \mathrm{CF} 88$ and CF24 and the type strains of recognized members of the genus Veillonella. Dialister pneumosintes ATCC $33048^{\top}$ was used as the outgroup organism. Accession numbers for 16S rRNA gene sequences are given for each strain. Bootstrap values are indicated at corresponding nodes. Bar, 0.01 substitutions per site.

dispar ATCC $17748^{\mathrm{T}}$, V. denticariosi CIP $109448^{\mathrm{T}}$, V. caviae DSM $20738^{\mathrm{T}}, V$. criceti ATCC $17747^{\mathrm{T}}$, V. ratti ATCC $17746^{\mathrm{T}}$, V. rodentium ATCC $17743^{\mathrm{T}}$ and $V$. montpellierensis ADV $281.99^{\mathrm{T}}$. Pseudoalteromonas atlantica T6c was used as the outgroup organism. The six novel strains formed a distinct taxon with robust bootstrap support $(99 \%)$ in the $r p o B$ gene tree (Fig. 2). When the interior branch test of phylogeny was employed to test the significance of the branch containing the novel strains, the confidence probability obtained with the neighbour-joining and minimum-evolution methods was $99 \%$, indicating robust discrimination between $V$. parvula and the six novel strains. Mean $r p o B$ gene sequence similarity among the six novel strains was $99.0 \%$ and was $98.4 \%$ among $V$. parvula strains, indicating homogeneity of the two taxa. Mean $r p o B$ gene sequence similarity between the novel strains and strains belonging to $V$. parvula, the most closely related species, was $91 \%$, indicating that strains $\mathrm{CF} 100^{\mathrm{T}}$, CF05, CF30, CF84, CF88 and CF24 represent a novel species. We suggest that as $16 \mathrm{~S}$ rRNA gene sequence comparisons are not suitable for differentiating between all members of the genus Veillonella, comparisons based on $r p o B$ gene sequences represent a valid method for species characterization. This approach is validated given that $V$. dispar, $V$. parvula and $V$. denticariosi cannot be separated satisfactorily by using $16 \mathrm{~S}$ rRNA gene sequence comparisons but can be defined clearly when $r p o B$ gene sequence data are considered (Fig. 2).

As strains $\mathrm{CF} 100^{\mathrm{T}}, \mathrm{CF} 05, \mathrm{CF} 30, \mathrm{CF} 84, \mathrm{CF} 88$ and $\mathrm{CF} 24$ formed a phylogenetically distinct group based on $r р о B$ gene sequencing, with robust bootstrap support, separate from V. parvula and all other recognized Veillonella species, we suggest that they represent a novel species of the genus Veillonella. We undertook a more complete phenotypic analysis of these strains.

Strains CF100 ${ }^{\mathrm{T}}$, CF05, CF30, CF84, CF88 and CF24 were tested, at least twice, by using the rapid ID 32A and API 20A identification kits (bioMérieux). Cells were small, Gram-negative coccoids, mainly appearing singly but with

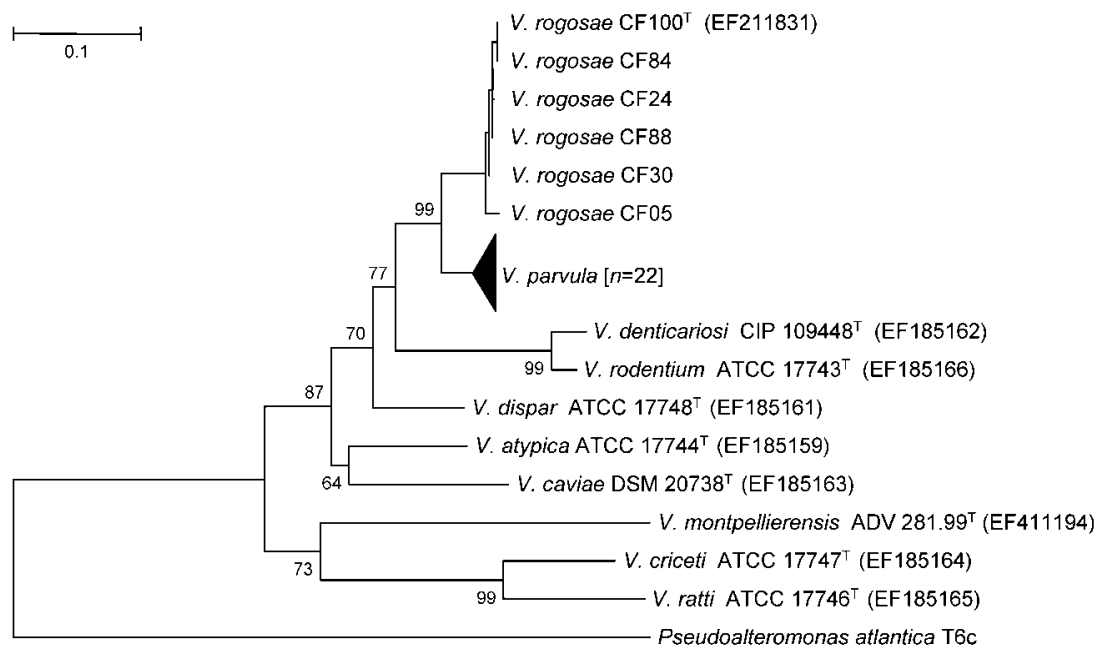

Fig. 2. Neighbour-joining tree based on partial $r p o B$ gene sequences (608 nt), showing relationships between strains $\mathrm{CF} 100^{\top}, \mathrm{CF} 05$, CF30, CF84, CF88 and CF24 and recognized members of the genus Veillonella. Pseudoalteromonas atlantica T6c (gi:109698613: 682317-686345) was used as the outgroup organism. The strains of $V$. parvula are not listed but were $V$. parvula ATCC $10790^{\top}$ (EF185158), V. parvula ATCC 17745 (EF185160) and an additional 22 rpoB gene sequences with accession numbers EU077574-EU077595. Bootstrap values are indicated at corresponding nodes. Bar, 0.1 substitutions per site. 
some short chains visible. Spores were not formed. No sugars were fermented in either of the identification kits. All strains were catalase-negative and did not hydrolyse aesculin, but one strain (CF84) weakly hydrolysed arginine. All isolates reduced nitrate. All were positive for pyroglutamic acid arylamidase and alkaline phosphatase was produced weakly by three of the six strains; all other test results were negative. No carbohydrates were fermented to acids in either test system. All strains fermented lactate, but not glucose, in peptone/yeast extract broth to produce major amounts of both propionic and acetic acids as the only acid end products when culture supernatants were analysed by using a GLC methodology (Delwiche et al., 1985). None of the six strains was positive for any of the following enzyme activities (Beighton et al., 1991) as measured by using 4-methyl umbelliferyl-linked fluorogenic substrates: $\alpha$-galactosidase, $\beta$-galactosidase, $\alpha$-glucosidase, $\beta$ glucosidase, $\beta$ - $N$-acetylgalactosaminidase, $\alpha$-arabinosidase, $\beta$ - $N$-acetylglucosaminidase, sialidase, $\alpha$-fucosidase or $\beta$ fucosidase. The strains did not exhibit cytochrome oxidase activity. As with the recently described species $V$. montpellierensis (Jumas-Bilak et al., 2004), and other species of the genus (Kolenbrander \& Moore, 1992), phenotypic characteristics were not sufficient to differentiate the novel isolates from other members of the genus.

\section{Description of Veillonella rogosae sp. nov.}

Veillonella rogosae (ro.go.sae. N.L. masc. gen. n. rogosae of Rogosa, named in honour of the late American microbiologist Morrison Rogosa, for his outstanding contributions to microbiology and to the taxonomy of the genus Veillonella).

Cells are Gram-negative, non-motile, non-sporulating coccoids $(0.3-0.5 \mu \mathrm{m}$ in diameter) that occur singly and in short chains. Colonies on Veillonella agar (Rogosa, 1956) are $2-4 \mathrm{~mm}$ in diameter and domed with an entire edge. Strictly anaerobic. Able to reduce nitrate, but unable to hydrolyse aesculin or arginine. Oxidase-negative. Unable to produce acids from carbohydrates and does not exhibit extracellular glycosidic enzyme activities. Exhibits pyroglutamic acid arylamidase activity and variable alkaline phosphatase activity. Major acid end products are acetic and propionic acids. Can be differentiated from other species of the genus Veillonella based on partial 16S rRNA and $r p o B$ gene sequencing.

The type strain, CF $100^{\mathrm{T}}\left(=\right.$ CCUG $54233^{\mathrm{T}}=$ DSM $\left.18960^{\mathrm{T}}\right)$, was isolated from supra-gingival plaque of a child. Strains CF05, CF30, CF84, CF88 and CF24, isolated from similar sources, are also included in the species.

\section{Acknowledgements}

This research was supported by The Dental Institute of King's College London and in part by the Wellcome Trust (grant number
076381). We thank Julie Downes for performing the acid end-product analysis.

\section{References}

Beighton, D., Hardie, J. M. \& Whiley, R. A. (1991). A scheme for the identification of viridans streptococci. J Med Microbiol 35, 367-372.

Byun, R., Carlier, J.-P., Jacques, N. A., Marchandin, H. \& Hunter, N. (2007). Veillonella denticariosi sp. nov., isolated from human carious dentine. Int J Syst Evol Microbiol 57, 2844-2848.

Delwiche, E. A., Pestka, J. J. \& Tortorello, M. L. (1985). The veillonellae: gram-negative cocci with a unique physiology. Annu Rev Microbiol 39, 175-193.

Jumas-Bilak, E., Carlier, J. P., Jean-Pierre, H., Teyssier, C., Gay, B., Campos, J. \& Marchandin, H. (2004). Veillonella montpellierensis sp. nov., a novel, anaerobic, Gram-negative coccus isolated from human clinical samples. Int J Syst Evol Microbiol 54, 1311-1316.

Kolenbrander, P. E. \& Moore, L. V. H. (1992). The genus Veillonella. In The Prokaryotes, 2nd edn, pp. 2034-2047. Edited by A. Balows, H. Trüper, M. Dworkin, W. Harder \& K.-H. Schleifer. New York: Springer.

Kumar, S., Tamura, K. \& Nei, M. (2004). MEGA3: integrated software for molecular evolutionary genetics analysis and sequence alignment. Brief Bioinform 5, 150-163.

Lane, D. J. (1991). 16S/23S rRNA sequencing. In Nucleic Acid Techniques in Bacterial Systematics, pp. 115-175. Edited by E. Stackebrandt \& M. Goodfellow. Chichester: Wiley.

Marchandin, H., Jean-Pierre, H., Carrière, C., Canovas, F., Darbas, H. \& Jumas-Bilak, E. (2001). Prosthetic joint infection due to Veillonella dispar. Eur J Clin Microbiol Infect Dis 20, 340-342.

Marchandin, H., Teyssier, C., Simeon De Buochberg, M., JeanPierre, H., Carriere, C. \& Jumas-Bilak, E. (2003). Intra-chromosomal heterogeneity between the four 16S rRNA gene copies in the genus Veillonella: implications for phylogeny and taxonomy. Microbiology 149, 1493-1501.

Marchandin, H., Teyssier, C., Jumas-Bilak, E., Robert, M., Artigues, A. C. \& Jean-Pierre, H. (2005). Molecular identification of the first human isolate belonging to the Veillonella ratti-Veillonella criceti group based on $16 \mathrm{~S}$ rRNA and dnaK gene sequencing. Res Microbiol 156, 603-607.

Mays, T. D., Holdeman, L. V., Moore, W. E. C., Rogosa, M. \& Johnson, J. L. (1982). Taxonomy of the genus Veillonella Prévot. Int J Syst Bacteriol 32, 28-36.

Rogosa, M. (1956). A selective medium for the isolation and enumeration of the veillonella from the oral cavity. J Bacteriol 72, 533-536.

Rogosa, M. (1984). Anaerobic Gram-negative cocci. In Bergey's Manual of Systematic Bacteriology, vol. 1, pp. 680-685. Edited by N. R. Krieg \& J. G. Holt. Baltimore: Williams \& Wilkins.

Saitou, N. \& Nei, M. (1987). The neighbor-joining method: a new method for reconstructing phylogenetic trees. Mol Biol Evol 4, 406-425.

Thompson, J. D., Higgins, D. G. \& Gibson, T. J. (1994). CLUSTAL W: improving the sensitivity of progressive multiple sequence alignment through sequence weighting, position-specific gap penalties and weight matrix choice. Nucleic Acids Res 22, 4673-4680. 